\section{Time of symptom onset and histologic findings in bladder pain syndrome/interstitial cystitis: new findings on useful correlations}

\author{
Daniele Porru, ${ }^{1}$ Valentina Bobbi, ${ }^{1}$ \\ Carmelo Di Franco, ${ }^{1}$ Alessandra Viglio, ${ }^{2}$ \\ Mattia Novario, ${ }^{2}$ Carmine Tinelli, ${ }^{3}$ \\ Barbara Gardella, ${ }^{4}$ Rossella Nappi, ${ }^{5}$ \\ Arsenio Spinillo, ${ }^{4}$ Marco Paulli, ${ }^{2}$ \\ Bruno Rovereto ${ }^{1}$
}

${ }^{1}$ Department of Urology; ${ }^{2}$ Department of Molecular Medicine, Pathology Unit;

${ }^{3}$ Clinical Epidemiology and Biometric

Unit; ${ }^{4}$ Obstetric and Gynecological

Clinic; ${ }^{5}$ Department of Clinical,

Surgical, Diagnostic and Pediatric

Sciences, Research Center of

Reproductive Medicine, Unit of

Gynecological Endocrinology and

Menopause, Fondazione IRCCS

Policlinico San Matteo, Pavia, Italy

\begin{abstract}
The aim was to find out whether a correlation exists between denudation of urothelium and time of symptom onset in patients with bladder pain syndrome/interstitial cystitis (BPS/IC), and to search a correlation between impact of symptoms evaluated with ICSI-ICPI and the presence of comorbid conditions associated with BPS/IC. Ninety-seven consecutive patients underwent cystoscopy under general anesthesia to classify those cases suspected of being affected from BPS/IC. Three cold bladder biopsies were taken including detrusor muscle. Statistical analysis showed significant correlation between IC/BPS duration and the presence of Hunner's lesions $(\mathrm{P}<0.023)$. Hunner's lesion with cystoscopy and histological evidence of urothelial denudation with bladder biopsy appear to be items related to IC/BPS duration. Thus an early diagnosis allows to start an appropriate therapeutic approach and prevent a more severe evolution of this multifaceted painful syndrome. Our study shows a correlation between time of symptom onset and evidence of urothelial denudation and with detrusor mast cell count in the whole group of patients. IC/BPS duration seem to correlate with the presence of associated diseases.
\end{abstract}

\section{Introduction}

The European Society for the Study of Interstitial Cystitis (ESSIC) defines bladder pain syndrome (BPS) as chronic (over 6 months) pelvic pain, pressure, or discomfort perceived to be related to the urinary bladder, associated with at least one other urinary symptom such as persistent urge to void or frequency in the absence of an identifiable cause. ${ }^{1}$

The exact prevalence of BPS is unclear, some authors assume that so far it has been widely underestimated..$^{2-5}$ The precise pathophysiological mechanisms of BPS remain unclear, although it has been hypothesized that IC/BPS could be pathophysiologically related to a disruption of the bladder mucosa surface layer, which causes loss of glycosaminoglycans (GAGs), a class of mucopolysaccharides with hydrorepellent properties, exposing the urothelium to various urinary toxic agents, which might cause disease progression.

Current understanding of the disease links abnormal differentiation of the urothelium, neural upregulation and increased presence of mast cells ${ }^{6-8}$ to its development.

Some clinicians now consider that many of the pathological features usually described are non-specific. ${ }^{9-11}$ However, intrafascicular fibrosis, an increase in the number of mast cells in the detrusor, denudation of urothelium in patients with a diagnosis of with BPS/IC are observations described in many studies. ${ }^{11}$ There are conflicting data on the usefulness of mast cell count to diagnose IC. The time elapsed between onset of symptoms and diagnosis was defined as time of symptom onset or IC/BPS duration, and since treatment is aimed at restoring bladder urothelial GAG layer in this infrequent, chronic and sometimes slowly progressive disease, we tried to examine whether a delay of disease identification can affect the results of treatment. The next step would be to speculate whether there is a relationship between urothelial erosion and Hunner's lesion. Although the number of cases with Hunner's lesions observed in our series was small, the issue seems challenging although controversial: could endoscopic and histologic features be a results of a long-standing urothelial inflammation from different causes, but with a common pathogenetic thread?

The primary aim of the study was to find out whether a correlation exists between denudation of urothelium and time of symptom onset in patients with BPS/IC.
Correspondence: Daniele Porru, Department of Urology, Fondazione IRCCS Policlinico San Matteo, via Campeggi, 27100 Pavia, Italy. Tel.: +39.0382.503872/503543 -

Fax: +39.0382 .503403 .

E-mail: danieleporru@tin.it

Key words: Interstitial cystitis; bladder pain syndrome; bladder biopsy; chronic pelvic pain.

Contributions: DP, VB, conception and design; AV, CDF, MN, CT, BG, RN, drafting and revising the article critically for important intellectual content; MP, AS, BR, final approval of the version to be published. All authors read and approved the manuscript.

Informed consent: Informed consent was obtained from all individual participants included in the study.

Registered trial: ClinicalTrials.gov: ID NCT03069053.

Conflict of interest: the authors declare no conflict of interest.

Received for publication: 20 June 2017

Revision received: 18 September 2017.

Accepted for publication: 2 October 2017.

This work is licensed under a Creative Commons Attribution NonCommercial 4.0 License (CC BY-NC 4.0).

(C) Copyright D. Porru et al., 2017

Licensee PAGEPress, Italy

Urogynaecologia 2017; 30:201

doi:10.4081/uij.2017.201

Since IC/BPS can be presently viewed as a complex general syndrome, ${ }^{12,13}$ secondary aim was to search a correlation between impact of symptoms evaluated with ICSIICPI and number of comorbid conditions associated with BPS-IC, which may appear in the years after the onset of the disease.

\section{Materials and Methods}

Between 2005 and 2016, in our Department 97 consecutive patients underwent cystoscopy under anesthesia to classify those cases suspected of BPS/IC. Patients reported long-lasting irritative bladder symptoms, including urgency, daytime frequency of seven or more episodes, nocturia, and pain in the lower abdomen, lower back, vagina or perineum during filling or after 
micturition. Alternative diagnoses such as urinary tract infection (UTI) were excluded. Confusable diseases were ruled out by thorough history, clinical examination, urine analysis and imaging.

In this retrospective study by means of a detailed medical history we analyzed the time of symptom onset, frequency of micturitions evaluated by bladder diary, the presence of associated diseases. All patients were given the same questionnaire (O'Leary-Sant Interstitial Cystitis Symptom and Problem Index), ${ }^{14}$ filled at the time of bladder biopsy, and 24 weeks after starting the same oral and intravesical treatment.

As for the evaluation of the ICSI-ICPI scores we considered relevant values those $\geq 12$, that is patients with severe symptoms. ${ }^{14,15}$

Patients suspected of having BPS-IC underwent cystoscopy with hydrodistension under general anesthesia. A rigid cystoscope with a Ch. 22 caliber was used, mannitol solution was used as filling fluid, and infusion height was $80 \mathrm{~cm}$ above the symphysis pubis. During filling the bladder was continuously inspected. At maximal capacity, distension was maintained until spontaneous filling stopped and maximum capacity was reached. At this point the bladder was drained and bladder walls and mucosa were closely examined. The bladder was then filled again at approximately half capacity, and at least three deep biopsies were taken, including detrusor muscle, from the anterior, posterior and lateral bladder walls. The biopsy was performed in order to obtain a pathological assessment and analyze the presence and grade of inflammation of bladder wall.

All biopsies were then fixed in 4\% formalin and sent to the Pathologist for examination. It was specifically asked to assess the presence of urothelial denudation, that is interruption/absence of urothelial layer of bioptic specimen, inflammatory infiltrate, counts of mast cells in the submucosa and detrusor, presence of intrafascicular fibrosis and submucosal bleeding. A cut-off of 27mast cells $/ \mathrm{mm}^{2}$ was used for the assessment of detrusor mastocytosis.

\section{Morphologic study}

Biopsy samples were cut in 5 - $\mu$ m-thick sections, then stained with the following reagents for morphologic evaluation: Hematoxylin-Eosin, Giemsa and Masson Tricromic Stain. This procedure was performed by automatic stainers (Leica, mod. ST5020 and mod. Autostainer ${ }^{\mathrm{TM}}$ XL).

If the biopsy wasn't adequately sized $\left(<1 \mathrm{~mm}^{2}\right.$-wide), we decided to evaluate the most reliable parameters, giving a N.V. (not valuable) response to the others.
Such parameters were single-blindly studied by two independent pathologists using optic microscopes (Nikon, mod. Eclipse $^{\mathrm{TM}} \mathrm{E} 400$ and mod. Eclipse ${ }^{\mathrm{TM}} \mathrm{CI}$ ) and discordant cases were then discussed jointly.

\section{Immunohistochemical studies}

Immunohistochemical reactions with Dako monoclonal antibody anti-Human Mast-cell Tryptase (Clone AA1; IgG1, Kappa), at 1:4000 dilution, were performed in order to highlight the mast-cell component of the inflammatory infiltrate, and then to quantify it. According to the protocol followed at the Laboratory of Immunohistochemistry of the Pathology Unit the automatic immunostainer Dako Omnis $^{\mathrm{TM}}$ was used.

\section{Evaluation of the immunostainings}

The reactions were evaluated using optic microscopes (Nikon, mod. Eclipse ${ }^{\mathrm{TM}}$ E400 and mod. Eclipse ${ }^{\mathrm{TM}} \mathrm{CI}$ ). In each biopsy the following parameters were studied, according to the recommendations reported by Hanno et al.: ${ }^{16}$ i) number of mastcells $/ \mathrm{mm}^{2}$ of mucosa; ii) number of mastcells $/ \mathrm{mm}^{2}$ of submucosa; iii) number of mast-cells $/ \mathrm{mm}^{2}$ of the detrusor layer.

In order to obtain the highest accuracy, a single-blind evaluation was performed by two independent pathologists; discordant or uncertain cases were then discussed jointly.

Three patients had complications in the whole group, one acute urinary retention after catheter removal, and two urinary tract infections treated successfully with a course of antibiotic treatment.

\section{Statistical analysis}

The Shapiro-Wilk test was used to test the normal distribution of quantitative variables; since they were all not normally distributed, the results were expressed as median and interquartile range (IQR; $25^{\text {th }}-75^{\text {th }}$ percentile). Qualitative variables were summarized as counts and percentages.

Mann-Whitney U test was used to analyze differences between two groups, while ANOVA non-parametric Kruskal-Wallis Test (with Bonferroni correction for multiple corrections) was used for more than two groups. Qualitative variables were summarized as counts and percentages and differences were analyzed with chi square test. Spearman's correlation coefficient was used to test the association between two quantitative continuous variables. The correlations were categorized as follows: 0.00 to 0.25 none or slight; 0.25 to 0.50 fair to moderate; 0.50 to 0.75 moderate to good; 0.75 to 1.00 almost perfect (Colton T, ed. Statistics in medicine. Boston MA: Little, Brown and Company; 1974). $\mathrm{P}<0.05$ was considered statistically significant. All tests were twosided. The data analysis was been performed with the STATA statistical package (release 13.1, 2014, Stata Corporation, College Station, Texas, USA).

\section{Results}

In this retrospective study we evaluated 97 women, all Caucasian. The average age at the time of biopsy was 39 years (range 19-67 years). The average time interval between the onset of symptoms and the diagnosis of IC/BPS at the time of biopsy was 7 years (range 1-38 years). Patients received various medical nonspecific treatments before a diagnosis was made.

Based on the results of the questionnaire, ICSI and ICPI scores were $>12$. The average voiding frequency was 17 voids/24 h (range 7-60). Cystoscopy with hydrodistention was normal in 10 patients $(9.7 \%)$, while in 9 patients $(9.2 \%)$ a Hunner's lesion was observed at cystoscopy before hydrodistension. Glomerulations grade II and III were found in 74 patients $(76.3 \%)$. Statistical analysis showed that the time of symptom onset in patients with HIC was longer than in patients with NHIC $(\mathrm{P}<0.023)$ (Figure 1).

A correlation between IC/BPS duration and the presence of glomerulations could not be found.

Histological features detected with bladder biopsies showed urothelial interruption of urothelium (urothelial denudation) in 75 patients, in only 7 patients detrusor mastocytosis was found $(>27$ mast cells $/ \mathrm{mm}^{2}$ ). This is the number of mast cells, which has been considered significant for detrusor mastocitosis. ${ }^{17}$

The analysis of these data showed a statistically significant correlation between the IC/BPS duration and urothelial denudation $(\mathrm{P}<0.029)$ (Figure 2); the same correlation

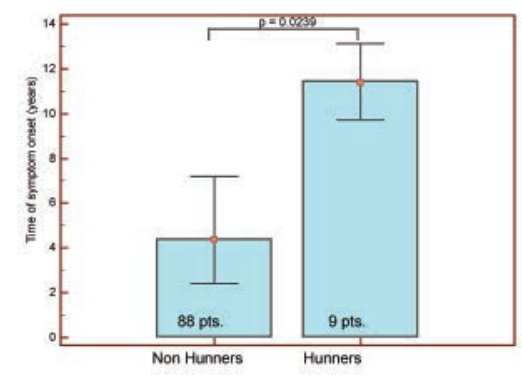

Figure 1. Time of symptom onset in Hunners interstitial cystitis and nonHunners. 
was also found $(\mathrm{P}=0.29)$ with detrusor mastocytosis $\left(>27\right.$ mast cells $\left./ \mathrm{mm}^{2}\right)$, although this feature could be found only in 7 out of 97 patients. It could be argued that mucosal loss in the specimens might be expected since they were taken after hydrodistention, where mucosal cracking is possible, as Johansson et al. reported, ${ }^{18}$ therefore we are not absolutely sure of the value of this finding. On the other hand, biopsy before hydrodistension should not performed due to the risk of fluid extravasation after the procedure.

No significant correlation was recorded between urothelial denudation and inflammatory infiltrate of detrusor mast cells.

Only one patient with severe symptoms proved refractory to conservative treatment and required major surgery, supratrigonal cystectomy. Table 1 reports the incidence of several associated conditions. The time of symptom onset did not seem to correlate with the severity of symptoms, but rather with the presence of diseases associated wit BPS/IC, such as fibromyalgia, vulvodynia, connective tissue diseases, irritable bowel syndrome and multiple allergies (Table 1). We found that patients with a higher number of comorbid conditions $(>2)$ also had a higher score of the ICSI-ICPI questionnaires, the correlation reached statistical significance $(\mathrm{P}=0.002)$ (Figure 3$)$. However IC/BPS duration was not found to be correlated with the severity of symptoms evaluated with ICSI-ICPI scores.

\section{Discussion}

Patients with interstitial cystitis are more likely to have undergone prior gynecologic surgery and/or to have a history of urinary tract infections and are 10-12 times more likely to report childhood bladder problems. ${ }^{19}$

During most of the twentieth century interstitial cystitis was diagnosed as a result of the cystoscopic detection of Hunner's ulcers.

Some clinical studies in the literature tried to clarify whether cystoscopy with hydrodistention was able to provide additional information to clinical evaluation. ${ }^{20,21}$ Ottem $^{22}$ compared a group of IC patients undergoing cystoscopy with hydrodistention with a control group, it was found a therapeutic result of the procedure, showing a decrease in the severity of symptoms in $56 \%$ which lasted, on average, about two months and found no difference in the course of the disease based on different age, time of symptom onset, urinary frequency and incidence of pelvic pain.

In our study we found that the time of symptom onset was related with the presence of urothelial denudation/erosion of urothelium found at histologic examination. Besides, all the small sample of patients with Hunner-type IC (HIC) had symptoms for more than 10 years.

Some authors believe Hunner-type/classic IC (HIC) and non-Hunner-type-interstitial cystitis (NHIC) are separate entities that differ in terms of clinicopathological features. ${ }^{23,24}$ Histologically, stromal infiltration of lymphocytes and plasma cells, edema, fibrosis, urothelial denudation and detrusor mastocytosis are usual histologic features of IC. ${ }^{25,26}$ Most of these findings are considered to be nonspecific/chronic inflammatory changes, besides thorough and consistent assessment of the degree of each histological alteration is quite difficult using common evaluation methods by means of semiquantitative analysis.

The results of some studies ${ }^{27}$ suggest that NHIC and HIC are distinct pathological entities, with the latter characterized by pancystitis, frequent clonal B-cell expansion and epithelial denudation. An abnormality in the B-cell population was found as a relevant feature in the pathogenesis of HIC. Bladder biopsy to confirm the diagnosis is a matter of controversy. Denson found that $30 \%$ of patients with BPS/IC had a normal histology. ${ }^{28}$ This percentage increases to $43 \%$ in the study performed by Matilla. ${ }^{29}$ Few well-structured studies correlate biopsy features with clinical findings and disease progression in IC, which led to question the role of bladder biopsy in the diagnosis of IC. ${ }^{30}$

Among the studies showing a correlation between clinical and histological findings Tomaszewski et al. revealed that the percentage of urothelial denudation and the percentage of submucosal hemorrhages was strongly associated with pain, as well as nocturia was strongly associated with mast cell density of the lamina propria and the presence of flogistic tissue of lamina propria. ${ }^{9}$

It has been reported ${ }^{28}$ that a significant loss of the epithelium was observed in HIC specimens. It is interesting that the epithelium of non-IC cystitis specimens was well preserved despite the presence of inflammatory cell infiltration. This could prompt to the conclusion that epithelial denudation is a specific alteration occurring in the urinary bladder of patients with HIC, and is not a result of chronic inflammation. We could then suppose that epithelial loss is the primary cause of HIC and stromal inflammation is a subsequent event. However, some special inflammatory process may take place in HIC that leads to epithelial denudation. It could be argued that mucosal loss in the specimens might be expected since they were taken after hydrodistention, where mucosal cracking is possible, therefore we are not absolutely sure of the value of this finding. On the other hand, biopsy before hydrodistension should not be performed due to the risk of fluid extravasation after the procedure.

There are also conflicting data on the usefulness of mast cell count to diagnose IC. Although some studies suggested that the presence of mast cells in the detrusor muscle is quite specific of IC, ${ }^{10,11}$ other data suggest that this cannot be confirmed. ${ }^{12,13}$

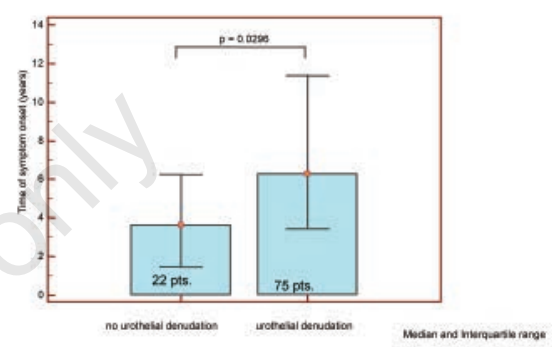

Figure 2. Correlation between time of symptom onset and denudation of urothelium/interruption of mucosa. No urothelial denudation: 22 patients; urothelial denudation: 75 patients.

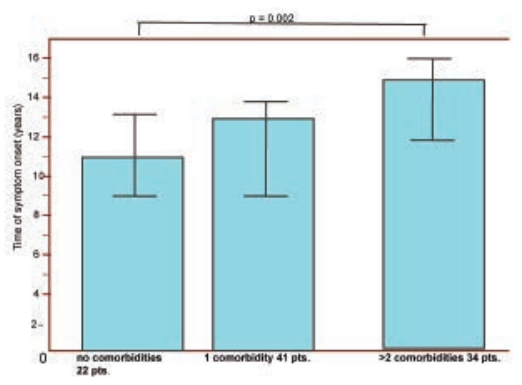

Figure 3. Correlation between interstitial cystitis/bladder pain syndrome duration and severity of symptoms (ICSI-ICPI questionnaires) in 97 patients (pts.).

Table 1. Comorbidities of patients.

\begin{tabular}{lc} 
Comorbidities & Incidence (\%) \\
Fibromyalgia & $29.80 \%$ \\
Vulvodynia & $21.00 \%$ \\
\hline Irritable bowel syndrome & $5.30 \%$ \\
Allergies & $8.80 \%$ \\
\hline Sjogren's syndrome & $3.50 \%$ \\
Endometriosis & $5.20 \%$ \\
\hline No associated pathologies & $26.40 \%$ \\
\hline
\end{tabular}


The importance of an early diagnosis for predicting the clinical evolution of the disease and the development of associated diseases of other organs or body sites has not yet been properly investigated. Our group of patients was not large enough to allow a comparison between HIC and NHIC patients, however our study showed a clear correlation between time of symptom onset and the evidence of urothelial denudation as well as with detrusor mast cell count in the whole group of HIC and NHIC patients. Interstitial cystitis is associated with a number of chronic illnesses, inflammatory bowel disease, systemic lupus erythematosus, irritable bowel syndrome, fibromyalgia, atopic allergy. Actually the pathophysiology of those conditions is similar to that of IC/BPS. ${ }^{24}$ Table 1 reports the percentage of associated conditions in our series.

\section{Conclusions}

It is difficult to predict the severity of the IC symptoms on the basis of bladder biopsy histology, however our findings lead to the hypothesis that the finding of urothelial denudation with histological examination of bladder biopsy and of Hunner's lesion with cystoscopy are items related to IC/BPS duration. Our findings therefore suggest something new, that is an early diagnosis could allow to begin as soon as possible the most appropriate therapeutic approach and prevent a more severe evolution of this multifaceted painful syndrome.

\section{References}

1. van de Merwe JP, Nordling J, Bouchelouche P. Diagnostic criteria, classification, and nomenclature for painful bladder syndrome/interstitial cystitis: an ESSIC proposal. Eur Urol 2008;53:60-7.

2. Fall M, Oberpenning F, Peeker R. Treatment of bladder pain syndrome/interstitial cystitis 2008: can we make evidencebased decisions? Eur Urol 2008;54:65-78.

3. van de Merwe JP, Nordling J, Bouchelouche P, et al. Diagnostic criteria, classification, and nomenclature for bladder pain syndrome/interstitial cystitis: an ESSIC proposal. Eur Urol 2008;53:60-7.

4. Wyndaele JJ, Van Dyck J, Toussaint N.
Cystoscopy and bladder biopsies in patients with bladder pain syndrome carried out following ESSIC guidelines. Scand J Urol Nephrol 2009;43:471-5.

5. Nordling J, Anjum FH, et al. Primary evaluation of patients suspected of having interstitial cystitis (IC). Eur Urol 2004;45:662-9

6. Larsen S, Thompson SA, Hald T, et al. Mast cells in interstitial cystitis. $\mathrm{Br} \mathrm{J}$ Urol 1982;54:283-6.

7. Hanno P, Levin RM, Monson FC, et al. Diagnosis of interstitial cystitis. J Urol 1990;143:278-81.

8. Theoharides TC, Kempuraj D, Sant GR. Mast cell involvement in interstitial cystitis: a review of human experimental evidence. Urology 2001;57:47-55.

9. Tomaszewski JE, Landis JR, Russack V, et al. Biopsy features are associated with primary symptoms in interstitial cystitis: results from the interstitial cystitis database study. Urology 2001;57:67-75.

10. Wang X, Liu W, O'Donnell M, et al. Evidence for the role of mast cells Iin cystitis-associated lower urinary tract dysfunction: a multidisciplinary approach to the study of cchronic pelvic pain research network animal model study. PLoS One 2016;11:e168772.

11. Epstein JI, Amin MB, Reuter VR. Bladder biopsy interpretation. Philadelphia: Lippincott, Williams \& Wilkins; 2004.

12. Rosamilia A, Igawa Y, Higashi S. Pathology of interstitial cystitis. Int $\mathrm{J}$ Urol 2003;10:S11-5.

13. Sant GR, Hanno PM. Interstitial cystitis: current issues and controversies in diagnosis. Urology 2001;57:82-8.

14. O'Leary MP, Sant GR, Fowler FJ Jr, et al. The interstitial cystitis symptom index and problem index. Urology 1997;49:58-63.

15. Inoue Y, Mita K, Kakehashi M, et al. Prevalence of painful bladder syndrome (PBS) symptoms in adult women in the general population in Japan. Neurourol Urodyn 2009;28:214-8.

16. Hanno P. Pathology. Interstitial cystitis/painful bladder. Chapt. 23. In: Hanno P, et al. Painful bladder syndrome (including interstitial cystitis); 2005. Available from: http://www.ics.org/publications/ici_3/v 2.pdf/chap23.pdf

17. Larsen MS, Mortensen S, Nordling J, Horn T. Quantifying mast cells in bladder pain syndrome by immunohistochemical analysis. BJU Int
2008;102:204-7.

18. Johansson SL, Fall M. Clinical features and spectrum of light microscopic changes in interstitial cystitis. J Urol 1990;143:1118-24.

19. D. Porru R, Politanò M, Gerardini GL, et al. Different clinical presentation of interstitial cystitis syndrome. Int Urogynecol J 2004;15:198-202.

20. Mouracade P, Lang H, Jacqmin D, Saussine C. Using the interstitial cystitis new diagnostic criteria in daily practice: about 156 patients. Prog Urol 2008;18:674-79.

21. Waxman JA, Sulak PJ, Kuehl TJ. Cystoscopic findings consistent with interstitial cystitis in normal women undergoing tubal ligation. J Urol 1998;160:1663-7.

22. Ottem DP, Carr LK, Perks AE. What is the value of cystoscopy with Hydrodistension for interstitial cystitis? Urology 2005;66:494-9.

23. Peeker R, Fall M. Toward a precise definition of interstitial cystitis: further evidence of differences in classic and nonulcer disease. J Urol 2002;167: 2470-2.

24. Fall M, Aldenborg F, Johansson S, Peeker R. Clinical characteristics support that interstitial cystitis is a heterogeneous syndrome. Urology 2001;57:129-30.

25. Fall M, Johansson SL, Aldenborg F. Chronic interstitial cystitis: a heterogeneous syndrome. J Urol 1987;137:35-8.

26. Lynes WL, Flynn SD, Shortliffe LD, Stamey TA. The histology of interstitial cystitis. Am J Surg Pathol 1990;14:96976.

27. Maeda D, Akiyama Y, Morikawa T, Kunita A. Hunner-type (classic) interstitial cystitis: a distinct inflammatory disorder characterized by pancystitis, with frequent expansion of clonal Bcells and epithelial denudation. PLoS One 2015 [Epub ahead of print].

28. Denson MA, Griebling MA, Cohen MB. Comparison of cystoscopic and histological findings in patients with suspected interstitial cystitis. J Urol 2000;164:1908-11.

29. Matilla J. Vascular immunopathology in interstitial cystitis. Clin Immunol Immunopathol 1992;143:648-55.

30. Denson MA, Griebling TL, Cohen MB. Comparison of cystoscopic and histological findings in patients with suspected interstitial cystitis. J Urol 2000;164:1908-11. 